\title{
Evaluación económica en salud y toma de decisiones en el contexto sanitario cubano
}

\section{Economic assessment in health care and the decision-making in the health care context}

\author{
DraC. Ana María Gálvez González, ${ }^{\mathrm{I}}$ DraC. Anaí Garcia Fariñas, ${ }^{\mathrm{I}}{ }^{\mathrm{MsC}}$. Carmen

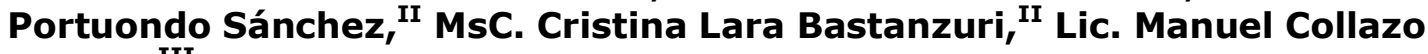 \\ Herrera ${ }^{\text {III }}$ \\ I Escuela Nacional de Salud Pública. La Habana, Cuba. \\ II Centro para el Desarrollo de la Farmacoepidemiología. La Habana, Cuba. \\ III Instituto Nacional de Higiene, Epidemiología y Microbiología (INHEM). La Habana, \\ Cuba.
}

\section{RESUMEN}

Objetivo: identificar cuáles son los factores que fortalecen y los que limitan el adecuado uso de las evaluaciones económicas en función de la toma de decisiones en el contexto sanitario cubano.

Métodos: se seleccionó un conjunto de profesionales vinculados a la toma de decisiones en diferentes ámbitos del Sistema Nacional de Salud. Se realizaron grupos focales para cada nivel identificado y entrevistas de profundidad a directivos, médicos, profesores e investigadores escogidos para conocer sus criterios al respecto.

Resultados: se encontraron factores de carácter práctico, metodológico y administrativo que fortalecen y otros que limitan el desarrollo de la evaluación económica en el país.

Conclusiones: se propone un conjunto de acciones para fortalecer el uso de la evaluación económica en salud en el proceso de toma de decisiones en Cuba.

Palabras clave: Evaluación económica, toma de decisiones, economía de la salud. 


\section{ABSTRACT}

Objective: to identify the factors that strengthen and those factors that limit the adequate use of economic assessment as a function of the decision-making process in the Cuban health care system.

Methods: there was selected a group of professionals linked to the decision-making process at various contexts of the national health care system. Focal groups for each identified level were organized in addition to making in-depth interviews to chosen managers, physicians, professors and researchers to find their criteria on this issue.

Results: practical, methodological and administrative factors that either strengthen or limit the development of economic assessment nationwide were identified. Conclusions: a number of actions were put forward to strengthen the use of economic assessment in health in the decision-making process in Cuba.

Key words: Economic assessment, decision-making, health economics.

\section{INTRODUCCIÓN}

La evaluación económica en salud como se ha planteado en otros espacios, ${ }^{1}$ constituye un instrumento valioso para la toma de decisiones. La creciente aparición de estudios en cursos, eventos y publicaciones científicas es un hecho que muestra una toma de conciencia en diferentes contextos acerca de lo anteriormente expresado.

Desde hace más de una década se ha fomentado la preocupación por el impacto de las evaluaciones económicas en la toma de decisiones. Se reconoce a Drummond, ${ }^{2}$ como el primero en verificar el alcance de la utilización de la evaluación económica en la toma de decisiones. Resultó interesante el proyecto "Euromet" desarrollado por la Universidad de Hannover donde se analizó el uso de los estudios de evaluaciones económicas como parte de la administración en salud en algunos países europeos. ${ }^{3}$ En España también se pueden encontrar antecedentes valiosos en el tema, como es el estudio conducido por Juárez Castelló y el análisis desarrollado por García Altés, entre otros. ${ }^{4,5}$

Durante el primer quinquenio de los años 2000 en América Latina se desarrolló el proyecto titulado "Desarrollo de Tecnologías de Evaluación Económica en América Latina" (NEVALAT), conducido por Joan Rovira, ${ }^{6}$ con la participaron 11 países, la mayor parte de América Latina, en el que Cuba estuvo presente. Este proyecto constituyó sin dudas un impulso para el desarrollo de la evaluación económica asociada a la toma de decisiones en salud en la región.

En Cuba se han realizado análisis sobre la situación y perspectivas de los estudios de evaluaciones económicas. ${ }^{7}$ Se han dado pasos para extender su utilización como mecanismo formal de evaluaciones de tecnologías sanitarias en aras de ganar en homogeneidad y transparencia de criterios, así como garantizar la posibilidad de reproducir los estudios que se presentan. ${ }^{8,9}$

El crecimiento de las evaluaciones económicas completas en los últimos cinco años en Cuba, se ha producido a expensas de la producción científica en el ámbito

http://scielo.sld.cu 
docente e investigativo. Estos estudios en mayor o menor medida han dado solución a problemas vinculados con la práctica sanitaria. Sin embargo, la presentación de estudios de evaluación económica directamente asociados al análisis de tecnologías sanitarias en instituciones de salud sigue siendo escasa. Todavía en el país no se exige la presentación formal de evaluaciones económicas por parte de las autoridades responsables de la toma de decisiones para la introducción de las nuevas tecnologías.

El presente estudio tiene el propósito de identificar cuáles son los factores que fortalecen y los que limitan el adecuado uso de las evaluaciones económicas en función de la toma de decisiones, en el contexto sanitario cubano.

\section{MÉTODOS}

Se seleccionó un conjunto de 83 profesionales vinculados a la toma de decisiones en diferentes niveles del Sistema Nacional de Salud, todos estuvieron relacionados, a través de la docencia y la investigación, al Departamento de Economía de la Salud de la Escuela Nacional de Salud Pública de Cuba durante el curso 2009-2010.

Estos profesionales se organizaron en cuatro grupos atendiendo a su relación con la toma de decisiones y lugar en el que se desempeñaban:

- Grupo 1. Directivos del Ministerio de Salud Pública de las áreas de economía y asistencia médica.

- Grupo 2. Directivos de hospitales, institutos, policlínicos y clínicas estomatológicas.

- Grupo 3. Médicos de familia, médicos de otras especialidades en hospitales y policlínicos, otros profesionales de la salud que trabajan en hospitales y policlínicos.

- Grupo 4. Profesores e investigadores de instituciones de salud.

Se realizaron grupos focales con cada uno de los grupos, con la finalidad de identificar los elementos que favorecen o los que frenan el uso de la evaluación económica, en el proceso de toma de decisiones. Se efectuaron entrevistas de profundidad a directivos, médicos, profesores e investigadores seleccionados de los grupos.

La información obtenida se clasificó siguiendo la experiencia desarrollada por Juárez Castelló y otros, ${ }^{4}$ basada en tres ejes, que son: aspectos de carácter administrativo, de orden metodológico y de orden práctico.

Para ampliar la información sobre los resultados de evaluaciones económicas en Cuba se consultó la base de datos de investigaciones y tesis, disponible en el departamento de Economía de la Salud de la Escuela Nacional de Salud Pública de Cuba (Economía de la Salud [CD-ROM]. Escuela Nacional de Salud Pública. CEDISAP).

Tanto en el desarrollo de los grupos focales como en las entrevistas realizadas se tuvo en cuenta los aspectos éticos que deben estar presentes en investigaciones de este tipo. 
El presente trabajo es un resultado parcial del tema "Evaluación Económica en Salud en Cuba. Desarrollos metodológicos" del ramal de Investigación en Sistemas y Servicios de Salud en Cuba.

\section{RESULTADOS}

La integración de los aspectos discutidos en los grupos focales con la información obtenida de las entrevistas de profundidad, permitió identificar un conjunto de elementos que promueven o frenan la utilización de la evaluación económica como instrumento para la toma de decisiones.

En las tablas 1 y 2 se presentan los resultados a partir de los ejes administrativos, metodológicos y prácticos. Se especifica el grupo de profesionales que los identificó.

Tabla 1. Factores que promueven el uso de la evaluación económica

\begin{tabular}{|c|c|c|}
\hline Eje de clasificación & & $\begin{array}{c}\text { Grupo } \\
\text { que los identificó }\end{array}$ \\
\hline \multirow{4}{*}{$\begin{array}{l}\text { De carácter } \\
\text { administrativo }\end{array}$} & $\begin{array}{l}\text { Voluntad política en la dirección } \\
\text { del SNS para realizar evaluaciones } \\
\text { económicas. }\end{array}$ & $1,2,3$ y 4 \\
\hline & $\begin{array}{l}\text { Directivos con formación básica } \\
\text { en temas económicos. }\end{array}$ & 1,2 y 4 \\
\hline & $\begin{array}{l}\text { Política sanitaria que promueve } \\
\text { el ahorro y la eficiencia en las acciones } \\
\text { de salud. }\end{array}$ & $1,2,3$ y 4 \\
\hline & $\begin{array}{l}\text { Presupuestos disponibles para asumir } \\
\text { acciones de salud. }\end{array}$ & 1,2 y 4 \\
\hline \multirow{5}{*}{$\begin{array}{l}\text { Relacionados } \\
\text { con el método }\end{array}$} & $\begin{array}{l}\text { Base de datos de costos publicada } \\
\text { por el área de economía del MINSAP. }\end{array}$ & 1 y 4 \\
\hline & $\begin{array}{l}\text { Disponibilidad de Guía Metodológica } \\
\text { para la Realización de Evaluaciones } \\
\text { Económicas en Salud. }\end{array}$ & $1,2,3$ y 4 \\
\hline & $\begin{array}{l}\text { Disponibilidad de guías de trabajo para } \\
\text { el uso racional de recursos sanitarios } \\
\text { en determinadas áreas, por ejemplo, } \\
\text { la de medicamentos. }\end{array}$ & $1,2,3$ y 4 \\
\hline & $\begin{array}{l}\text { Disponibilidad y acceso gratuito a la } \\
\text { formación en técnicas de evaluación } \\
\text { económica en salud. }\end{array}$ & 1 y 4 \\
\hline & $\begin{array}{l}\text { Grupos de trabajo multidisciplinarios } \\
\text { entrenados para la realización } \\
\text { de evaluaciones económicas. }\end{array}$ & 4 \\
\hline \multirow[t]{2}{*}{ De orden práctico } & $\begin{array}{l}\text { Directivos que estimulan en sus equipos } \\
\text { de trabajo realizar evaluaciones } \\
\text { económicas en las principales acciones } \\
\text { que se realizan en sus instituciones. }\end{array}$ & 1,2 y 4 \\
\hline & $\begin{array}{l}\text { Asignación de recursos y tiempo } \\
\text { para la realización de evaluaciones } \\
\text { económicas. }\end{array}$ & 1 y 4 \\
\hline
\end{tabular}

http://scielo.sld.cu 
Tabla 2. Factores que frenan el uso de la evaluación económica

\begin{tabular}{|c|c|c|}
\hline Eje de clasificación & & $\begin{array}{c}\text { Grupo } \\
\text { que los identificó }\end{array}$ \\
\hline \multirow{4}{*}{$\begin{array}{l}\text { De carácter } \\
\text { administrativo }\end{array}$} & $\begin{array}{l}\text { Se hace mayor énfasis en el control del } \\
\text { recurso que en su utilización eficiente. }\end{array}$ & 2 y 4 \\
\hline & $\begin{array}{l}\text { Presupuestos poco flexibles para } \\
\text { cambios en tecnologías. }\end{array}$ & 2 y 4 \\
\hline & $\begin{array}{l}\text { Con frecuencia se hace énfasis en } \\
\text { promover la tecnología de cada } \\
\text { institución sin otros análisis económicos } \\
\text { ni tener en cuenta que los recursos } \\
\text { son limitados. }\end{array}$ & 1 y 4 \\
\hline & $\begin{array}{l}\text { Aún no se tiene toda la certeza en } \\
\text { que los resultados de evaluaciones } \\
\text { económicas publicadas hayan sido } \\
\text { tenidos en cuenta en la toma de } \\
\text { decisiones. }\end{array}$ & 2,3 y 4 \\
\hline \multirow{5}{*}{$\begin{array}{l}\text { Relacionados con } \\
\text { el método }\end{array}$} & $\begin{array}{l}\text { Aunque existe la guía metodológica su } \\
\text { utilización se limita al nivel académico } \\
\text { y algunos centros de investigación. }\end{array}$ & 4 \\
\hline & $\begin{array}{l}\text { Dificultades para interpretar y aplicar } \\
\text { algunas técnicas y pasos que se utilizan } \\
\text { en las evaluaciones económicas. }\end{array}$ & $1,2,3$ y 4 \\
\hline & $\begin{array}{l}\text { Dificultades para generalizar los } \\
\text { resultados a diferentes contextos. }\end{array}$ & 4 \\
\hline & $\begin{array}{l}\text { Insuficientes equipos multidisciplinarios } \\
\text { entrenados en el país. }\end{array}$ & $1,2,3$ y 4 \\
\hline & $\begin{array}{l}\text { No se profundiza en el tratamiento } \\
\text { metodológico en temas sobre equidad } \\
\text { y calidad de vida. }\end{array}$ & 4 \\
\hline \multirow{4}{*}{ De orden práctico } & $\begin{array}{l}\text { Falta financiamiento para llevar a cabo } \\
\text { las evaluaciones económicas. }\end{array}$ & 1,2 y 4 \\
\hline & $\begin{array}{l}\text { No todos los implicados en el proceso } \\
\text { de toma de decisiones tienen } \\
\text { conciencia de la importancia de la } \\
\text { evaluación económica para la toma de } \\
\text { decisiones. }\end{array}$ & 2,3 y 4 \\
\hline & $\begin{array}{l}\text { Falta de continuidad en el seguimiento } \\
\text { de los resultados de los estudios. }\end{array}$ & 4 \\
\hline & $\begin{array}{l}\text { No se complementan las evaluaciones } \\
\text { económicas con otras herramientas } \\
\text { técnicas implementadas en la práctica } \\
\text { a fin de viabilizar la toma de } \\
\text { decisiones. }\end{array}$ & $1,2,3$ y 4 \\
\hline
\end{tabular}


En relación con los factores de carácter administrativo se puede apreciar tanto en la tabla 1 como en la 2 que los grupos 1,2 y 4 identificaron más fortalezas y debilidades. Todos los grupos coincidieron en que existe voluntad política y preocupación por desarrollar evaluaciones económicas en salud en aras de la eficiencia. Los grupos 2 y 4 identificaron la mayor parte de las limitaciones de carácter administrativo. Esto está relacionado directamente con que son los grupos en los que se desarrollan la mayor parte de las evaluaciones económicas.

Desde el punto de vista del método, los grupos 1 y 4 reflejaron la mayor parte de las los elementos que promueven y los que frenan las evaluaciones económicas. Todos los grupos le dieron valor a la existencia de la guía metodológica para la realización de evaluaciones económicas, sin embargo integrantes del grupo 4 fueron los que plantearon la necesidad de hacer uso sistemático de la misma. Este mismo grupo 4 identificó la mayor parte de las limitaciones. Todos los grupos identificaron la falta de equipos multidisciplinarios para llevar a cabo las evaluaciones económicas y la dificultad para comprender y aplicar todas las técnicas y herramientas que acompañan a un estudio de este tipo.

El grupo 4 mostró un mayor dominio de temas de mayor complejidad en las evaluaciones económicas como son, el problema de la generalización y del tratamiento de la equidad y la calidad de vida.

Respecto a los factores de carácter práctico resultó interesante que todos los grupos identificaron como una fortaleza la disponibilidad de guías prácticas de trabajo que conducen al uso racional de los recursos, por ejemplo, la disponibilidad del cuadro básico de medicamentos, ${ }^{10}$ o su enfoque regulador. ${ }^{11}$

Como debilidades todos los grupos identificaron la falta de personal dedicado a la realización de evaluaciones económicas y que sus resultados no se complementan con otras técnicas, por ejemplo, las de impacto presupuestario. ${ }^{12}$ Resultó interesante la preocupación manifestada respecto a disponer de evidencias de la utilización, para la toma de decisiones, de las evaluaciones económicas realizadas.

\section{DISCUSIÓN}

La preocupación creciente por analizar los factores que favorecen o limitan el desarrollo de las evaluaciones económicas es un hecho presente en diferentes contextos sanitarios. ${ }^{13-17}$ Sin embargo, el nivel de desarrollo alcanzado por cada país es diferente, algunos conducen procesos más consolidados, otros se encuentran en etapas preliminares. En cualquier caso, todos tienen retos pues el utilizar las evaluaciones económicas en el proceso de toma de decisiones no es un camino recto y libre de dificultades.

En Cuba, la utilización de la evaluación económica como instrumento para la toma de decisiones ha mostrado avances (generalmente a partir de tesis) en estos últimos años. Este auge no solamente se aprecia en el incremento de los estudios realizados, sino en el establecimiento y priorización de políticas y una creciente cultura dirigida al uso racional y eficiente de los recursos. ${ }^{18}$

Algunos de los resultados de este estudio coinciden con otros realizados en países de más experiencia en el tema. ${ }^{19-21}$ Entre estos se pueden citar la promoción de una cultura de evaluación, existencia de una guía metodológica para la realización de evaluaciones económicas adaptada al contexto y programas de formación en el 
tema. De la misma forma coinciden algunos retos como son, la necesidad de fomentar las prácticas de efectividad mostrada. Es cierto que su ausencia afecta la credibilidad de la eficiencia de la tecnología evaluada; lo que no es efectivo no puede ser eficiente. Otros elementos de coincidencia son la necesidad de puntualizar aspectos en las guías metodológicas disponibles, el peso de las evaluaciones concentradas en el área de medicamentos y menos desarrollo en las áreas de promoción y prevención de salud.

También existen coincidencias con otros países que aún se consideran en etapas más tempranas, ${ }^{22,23}$ (Valencia A. Contribuciones y limitaciones de la evaluación económica en la conformación del paquete básico de servicios en el sector salud. Salud Pública Méx. Año/vol.49, número especial. Instituto Nacional de Salud Pública. Cuernavaca. México. p. 236), por ejemplo, la necesidad de intensificar la formación de personal en estas técnicas, la insuficiencia de equipos multidisciplinarios con dominio en el tema y el escaso uso de las evaluaciones que se realizan para la toma de decisiones.

Quedan pendientes otros temas que también constituyen retos para muchos países, entre ellos, el hecho de que en las evaluaciones económicas que se realizan en Cuba, prácticamente no son abordados los aspectos relacionados con las mediciones de la calidad de vida. Esta ausencia puede generar dificultades en las decisiones entre intervenciones de diferentes problemas de salud con implicaciones severas sobre la equidad entre grupos de población.

Por último y no menos importante son los aspectos asociados a la generalización de resultados de las evaluaciones económicas. Este es uno de los retos más difíciles de enfrentar y que precisará el trabajo unido y sistemático de profesionales de diferentes contextos y realidades, más que un reto, hoy constituye un sueño.

A partir de las experiencias incorporadas en este estudio, se propone un conjunto de recomendaciones y tareas en aras de estimular y fortalecer el uso de la evaluación económica en el proceso de toma de decisiones en Cuba. Estas son las siguientes:

1. Publicar una nueva versión de la guía metodológica para las evaluaciones económicas donde se puntualicen temas que resultan de difícil comprensión para investigadores y encargados de la toma de decisiones. Realizar entrenamientos para el uso de esta guía.

2. Extender la formación de equipos multidisciplinarios que realicen evaluaciones económicas. Destacar el rol de directivos y técnicos en el proceso de evaluación económica y toma de decisiones.

3. Profundizar y actualizar en la incorporación de tecnologías y herramientas para la ejecución de evaluaciones económicas, por ejemplo, el uso de modelos matemáticos, nuevos modelos contables, aplicaciones informáticas, nuevas tecnologías de la información y las comunicaciones.

4. Estudiar variantes de flexibilización de presupuestos para facilitar la toma de decisiones.

5. Organizar equipos multidisciplinarios y con participación intersectorial con la finalidad de homogeneizar criterios en temas tales como tasas de cambio, descuentos en el tiempo, entre otros.

6. Implementar procesos de auditoria externa como vía de garantizar la transparencia en las propuestas de nuevas tecnologías. 
7. Que las tecnologías que se vayan a introducir lleven como anexo una evaluación económica que fundamente la decisión a adoptar.

8. Fomentar los estudios multicéntricos y la relación de intercambio con instituciones de mayor experiencia en el tema.

\section{REFERENCAS BIBLIOGRÁFICAS}

1. Oliva J, Antoñanzas F, Rivero Arias O. Evaluación económica y toma de decisiones en salud. El papel de la evaluación económica en la adopción y la difusión de tecnologías sanitarias. Informe SESPAS 2008. Gac Sanit. 2008;22(Supl 1):137-42.

2. Drummond MF, Cooke J, Walley T. Economic Evaluation under managed competition: evidence from the U.K. Social Science Medicine. 1997;45(4):583-95.

3. Graf von der Schulenburg JM, editors. The influence of economic evaluation studies on health care decision-making. Amsterdam: IOS Press; 2000.

4. Juárez $C$, Antoñanzas $F$. Impact of the studies of economic evaluation in health care decision-making. En: Castaño E, Antoñanzas F, Fuster J, editores. Avances en gestión sanitaria: implicaciones para la política, las organizaciones sanitarias y la práctica clínica. Barcelona: Asociación de Economía de la Salud; 2000. p. 141-50.

5. García Altés A. Twenty years of health economic analysis in Spain: are we doing well? En: Avances en la Gestión Sanitaria. Implicaciones para la política Sanitaria: Implicaciones para la política, las organizaciones sanitarias y la práctica clínica. XX Jornadas de Economía de la Salud; 2000 mayo 3-5. Palma de Mallorca, España: Asociación Española de Economía de la Salud; 2000.

6. Rovira J. Results of the Nevalat Project. Second Plenary session at the ISPOR first Latin American Conference; 2007 septiembre 9-11. Cartagena de Indias, Colombia: ISPOR; 2007.

7. Gálvez González AM, Álvarez Muñiz M, Sarduy Domínguez Y, Morales Laberón M. Diagnóstico de la evaluación económica en salud en Cuba. Rev Cubana Salud Pública [Internet]. 2004 [citado 30 de diciembre de 2011];30(1). Disponible en: http://scielo.sld.cu/scielo.php?script=sci arttext\&pid=S086434662004000100003\&lng=es\&nrm=iso\&t|ng=es

8. Gálvez González AM. Guía metodológica para la evaluación económica en salud: Cuba, 2003. Rev Cubana Salud Pública [Internet]. 2004 [citado 30 de diciembre de 2011];30(1). Disponible en:

http://scielo.sld.cu/scielo.php?script=sci arttext\&pid=S0864-

34662004000100005\&lng=es\&nrm=iso\&tlng=es

9. García Fariñas A, Gálvez González AM, García Rodríguez JF. Aspectos metodológicos críticos en las evaluaciones económicas de salud en el contexto cubano. Rev Cubana Salud Pública [Internet]. 2010 [citado 30 de diciembre de 2011];36(3). Disponible en:

http://scielo.sld.cu/scielo.php?script=sci arttext\&pid=S0864-

34662010000300007\&lng=es\&nrm=iso\&tIng=es

http://scielo.sld.cu 
10. Centro para el desarrollo de la Farmacoepidemiología. Cuadro Básico de Medicamentos [Internet]. 2010 [citado 30 de diciembre de 2011]. Disponible en: http://fnmedicamentos.sld.cu/

11. De la Cruz Pérez C, Pérez Cristiá R, Lara Bastanzuri C. Importación de medicamentos en Cuba: acceso, calidad y menor costo en el periodo 2004-2007. Anuario Científico. Año 6. Suplemento especial del Boletín INFO CECMED [Internet]. 2008 [citado 30 de diciembre de 2009]. Disponible en: http://www.cecmed.sld.cu/Docs/Pubs/Anuario/AC2009.pdf

12. Darbá J, Restovic G, Rejas J. Los modelos de impacto presupuestario y la toma de decisiones. Aplicación al trastorno bipolar tipo I en fase de manía aguda en España. Spanish Research Articles. Pharmacoeconomics. 2006;3(3):151-62.

13. Eddama O, Coast J. Use of economic evaluation in local health care decisionmaking in England: a qualitative investigation. Health Policy. 2009;89(3):261-70. Epub 2008 Jul 25.

14. Eddama O, Coast J. A systematic review of the use of economic evaluation in local decision-making. Health Policy. 2008;86(2-3):129-41. Epub 2008 Jan 14.

15. Hartz S, John J. Contribution of economic evaluation to decision making in early phases of product development: a methodological and empirical review. Int J Technol Assess Health Care. 2008;24(4):465-72.

16. Oliva J, Puig J, Bernal E. Evaluación Económica de Medicamentos: experiencias y vías de avance. Una visión complementaria. Gac Sanit. 2008;22(4):358-61.

17. Chaikeldkaew Chaikledkaew U, Lertpitakpong C, Teerawattananon Y, Thavorncharoensap M, Tangcharoensathien. The current capacity and future development of economic evaluation for policy decision making: a survey among researchers and decision-makers in Thailand. Value Health. 2009;12 (Suppl 3): S31-5.

18. Galvez González AM. Economía y salud en el camino hacia la eficiencia. Rev Cubana Salud Pública [Internet]. 2010 [citado 22 Oct 2010];36(1):1-2. Disponible en: http://scielo.sld.cu/scielo.php?script=sci arttext\&pid=S0864-

$34662010000100001 \& \operatorname{lng}=$ es\&nrm=iso\&tlng=es

19. Lessard C, Contandriopoulos AP, Beaulieu MD. The role of economic evaluation in the decision-making process of family physicians: design and methods of a qualitative embedded multiple-case study. BMC Family Practice [Internet]. 2009 [citado 22 Oct 2010];10:15. Disponible en: http://www.biomedcentral.com/1471$2296 / 10 / 15$

20. Van Velden ME, Severens JL, Novak A. Economic evaluations of health care programmes and decision making: the influence of economic evaluations on different healthcare decision-making levels. Pharmacoeconomics.

2005;23(11):1075-82.

21. López Bastida J, Oliva J, Antoñanzas F, García Altés A, Gisbert R, Mar J, Puig J. Spanish recommendations on economic evaluation of health technologies. J Eur J Health Economic. 2010;11(5):513-20. Epub 2010 Apr 20.

http://scielo.sld.cu 
22. Yothasamut J, Tantivess S, Teerawattananon Y. Using economic evaluation in policy decision-making in Asian countries: mission impossible or mission probable? Value Health. 2009;12(suppl 3):S26-30.

23. Iglesias CP, Drummond MF, Rovira J. NEVALAT Project Group. Health-care decision-making processes in Latin America: problems and prospects for the use of economic evaluation. Int J Technol Assess Health Care. 2005;21(1):1-1.

Recibido: 8 de diciembre de 2010.

Aprobado: 28 de noviembre de 2011.

Ana María Gálvez González. Escuela Nacional de Salud Pública. Calle 100 No. 1132 e/ E y Perla. Altahabana, Municipio Boyeros. La Habana, Cuba.

Correo electrónico: galveza@infomed.sld.cu, alastor@infomed.sld.cu 